\title{
MicroRNA-214-5p Inhibits the Invasion and Migration of Hepatocellular Carcinoma Cells by Targeting Wiskott-Aldrich Syndrome Like
}

\author{
Hongdan Li Haoqi Wang Zhen Ren \\ Life Science Institute, Jinzhou Medical University, Jinzhou, China
}

\section{Key Words}

MicroRNA-214-5p • Hepatocellular carcinoma $・$ Invasion $\bullet$ Migration $\bullet$ WASL

\begin{abstract}
Background/Aims: This study aims to explore the effects of microRNA-214-5p (miR-214-5p) on the invasion and migration of Hepatocellular Carcinoma cells (HCC). Methods: Hepatocellular Carcinoma tissues and adjacent normal tissues from 44 hepatocellular carcinoma patients were prepared for this study. The HepG2 and BEL-7402 cells were transfected with miR-214$5 p$ mimic and inhibitor. qRT-PCR was performed to detect the expressions of miR-214-5p. Transwell assays were used to detect the invasion and migration assays in HepG2 and BEL7402 cells. A dual-luciferase reporter assay was conducted to examine the effect of miR-214$5 p$ on Wiskott-Aldrich Syndrome Like (WASL/ N-WASP). Western blot and qRT-PCR were used to measure the expressions of the E-cadherin, N-cadherin and Vimentin proteins. Transwell chamber assays were performed to detect cell invasion and migration. Results: Compared with normal tissues, HCC tissues demonstrated significantly lower expression of miR-214-5p. Overexpression of miR-214-5p significantly inhibited the migration and invasion of HCC cells and inhibition of miR-214-5p promoted the migration and invasion. Additionally, miR-214-5p suppressed the epithelial-mesenchymal transition (EMT). Further study showed WASL was a putative target gene of miR-214-5p. Up-regulating the expression of WASL could reverse the inhibition effect of miR-214-5p on invasion and migration. Conclusion: Our data suggested that miR-214-5p inhibited the invasion and migration of HepG2 and BEL-7402 by targeting WASL in Hepatocellular carcinoma.

\section{Introduction}

Hepatocellular Carcinoma is the sixth most common cancer and the third-leading cause of cancer-related death worldwide [1]. Recently, the incidence of HCC has increased [2]. After curative resection [3, 4], HCC frequently breaks out tumor recurrence and metastasis

Li Hongdan

KARGER
Life Science Institute, Jinzhou Medical University, Section3

No.40, Songpo Road, Jinzhou, Liaoning 121000 (China)

Tel. +86-416-4673472 E-Mail lihongdan101@126.com 


\section{Cellular Physiology Cell Physiol Biochem 2018;46:757-764 \begin{tabular}{lll} 
DOI: 10.1159/000488734 & and Biochemistry & $\begin{array}{l}\text { O } 2018 \text { The Author(s). Published by S. Karger AG, Basel } \\
\text { wwww.karger.com/cpb }\end{array}$ \\
\cline { 2 - 3 }
\end{tabular}}

Li et al.: miR-214-5p Inhibits Hepatocellular Carcinoma

$[5,6]$. Although much effort has been made, there are no effective therapeutic strategy to cure Hepatocellular carcinoma [7, 8]. Therefore, it is of great significance to identify the mechanism of migration and invasion and develop targeted therapeutics.

N-WASP is a scaffold that links upstream signals to the activation of the Arp2/3 complex, leading to a burst of actin polymerization [9]. Cancer cell movement requires dynamic remodeling of the cytoskeleton and cell membrane and is controlled by multiprotein complexes including focal adhesion kinase (FAK) or the Neural Wiskott-Aldrich Syndrome Protein (N-WASP) [9]. MicroRNAs (miRNAs) are widely accepted to have a profound effect on gene expression, cellular homeostasis, and expressions of multiple oncogenes and tumor suppressor genes in cancer cells $[10,11]$. Modulating the functions of miRNAs might be effective in developing novel adjuvant therapeutics. MiR-214-5p is a product of the $110 \mathrm{bp}$ miR-214 gene in the intron of the Dynamin-3 gene on human Chromosome 1-NC_000001.10, which produces a mature miRNA with a sequence of ugccugucuacacuugcugugc [12]. In 2012, Iizuka $M$ and his collages revealed miR-214-5p may play crucial roles in the activation of stellate cells and the progression of liver fibrosis [13]. Moreover, in serum, Elemeery MN, et al. found miR-214-5p could be considered as early biomarkers for tracking the progress of liver fibrosis to HCC [14]. Zhang, et al. showed that miR-214-5p suppresses proliferation and invasion of human osteosarcoma cells by targeting ROCK1 [15]. Li et al. found in osteoblastic MC3T3-E1 cells, inhibition of microRNA-214-5p promotes survival and extracellular matrix formation [16]. Chandrasekaran et al. showed in human cervical and colorectal cancer cells, microRNA-214 suppresses growth, migration and invasion through AT-hook 1 [17]. Yang et al. [18] and Li et al. [19] also found miR-214 acts as a tumor suppressor in liver cancer proliferation and cell cycle. There also some different view, many researchers showed miR214 promoted tumor pathogenesis, such as in gastric cancer cell [20,21], ovarian cancer cells [22]. However, the roles of miR-214-5p on HCC invasion remain unknown.

Here, we report the upregulation of miR-214-5p inhibits the invasion and migration in HCC. Importantly, the functional effect of miR-214-5p is exerted by epithelial-mesenchymal transition (EMT). And miR-214-5p inhibited the invasion and migration by targeting WASL. Thus, our findings provide a novel mechanistic role for miR-214-5p in HCC metastasis and a rationale for clinically exploring the use of this microRNA in curbing HCC.

\section{Materials and Methods}

\section{Ethics statement}

This study was approved by the Ethics Committee of Jinzhou medical university. All samples were collected based on the informed consent of the study subjects.

\section{Cell culture}

Human HCC cells lines (HepG2 and BEL-7402) were purchased from the Institute of Biochemistry and Cell Biology, Shanghai Institutes for Biological Sciences, Chinese Academy of Sciences (Shanghai, China). The two cell lines were cultured in an incubator $\left(37^{\circ} \mathrm{C}, 5 \% \mathrm{CO}_{2}\right.$ and saturated humidity) with RPMI 1640 medium supplemented with $10 \%$ fetal bovine serum (FBS). The culture medium was replaced every two days. When the cells reached $90 \%$ confluence, they were passaged at a ratio of 1:4.

\section{Cell transfection}

For the cell transfection experiments, the cells were divided into the following groups: (1) miR214-5p mimics group (transfected with artificially synthesized miR-214-5p mimics); (2) miR-214-5p-NC group (transfected with a scrambled sequence); (3) miR-214-5p inhibitor (transfected with miR-214-5p inhibitors); (4) control group (no sequence transfected); (5) WASL group (transfected with WASL overexpression vector); (6) Vector group (transfected with empty plasmid vector); and (7) miR-214-5p mimics + WASL group (transfected with miR-214-5p mimics and WASL over-expression vector). The chemically synthesized miR-214-5p mimics, miR-214-5p inhibitors and negative control sequences were all purchased from Guangzhou RiboBio Co., Ltd., China. The cells were inoculated into a 6-well plate $24 \mathrm{~h}$ before 


\section{Cellular Physiology Cell Physiol Biochem 2018;46:757-764

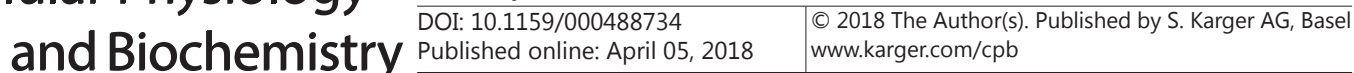

Li et al.: miR-214-5p Inhibits Hepatocellular Carcinoma

transfection. When the cells reached approximately 50\% confluence, the human HepG2 and BEL-7402 cell line was transfected using Lipofectamine2000 (Invitrogen Inc., Carlsbad, CA, USA). After 6 hours, the culture medium was replaced. After $48 \mathrm{~h}$ in culture, the cells were then collected for further experiments.

\section{Transwell chamber assay}

Cell migration and invasion were measured using 24-well Transwell plate cell culture inserts (Corning,Cambridge, MA, USA) with or without Matrigel coating (BD Biosciences, San Jose, CA, USA). Fortyeight hours after transfection, the HepG2 and BEL-7402 cells were collected for serum starvation, where the cells were cultured for $24 \mathrm{~h}$ in FBS-free RPMI 1640 cell culture medium. The collected cells were suspended in FBS-free RPMI 1640 cell culture medium, which was followed by adjustment of the cell concentration to $10^{5}$ cells $/ \mathrm{mL}$. The serum-free cell suspension $(200 \mu \mathrm{L})$ was added to the upper chamber of a Transwell invasion chamber, and RPMI 1640 culture medium containing 10\% FBS (600 $\mu \mathrm{L})$ was added to the lower chamber; the cells were then cultured for $48 \mathrm{~h}$ at $37^{\circ} \mathrm{C}$ with $5 \% \mathrm{CO}_{2}$. After the liquid in the upper chamber was absorbed, the cells that did not pass through the micropores on the membrane were removed with cotton swabs, which was followed by staining with $4 \%$ crystal violet for $10 \mathrm{~min}$. Then, cells that had passed through the membrane and infiltrated the lower chamber were then imaged under a microscope. After the dye on the membrane was dissolved with acetic acid, the cells were transferred to a 96-well plate. The absorbance value of each well at a wavelength of $570 \mathrm{~nm}$ was detected. The experiment was repeated 3 times for each group.

\section{Dual-luciferase reporter assay.}

Plasmids encoding a portion of the 3'-untranslated region (3'UTR) of WASL linked to the firefly luciferase protein. Firefly luciferase constructs were co-transfected with Renilla luciferase vector control (TK) into HepG2 cells. Where indicated, HepG2 cells were stably expressing miR-214-5p. Twenty-four hours after co-transfection with 3'UTR of target gene and TK (ratio 1:10), HepG2 cells were detached, washed, and dissolved in passive lysis solution (PLS) in $15 \mathrm{~min}$, at room temperature. Luciferase activities were measured consecutively (Dual-Luciferase Assay; Promega), and the relative luciferase activity was assessed: (firefly activity)/ (Renilla activity).

\section{RNA isolation and qRT-PCR}

Total RNA was prepared from tissues or cells using TRIzol reagent (TaKaRa, Dalian, China). cDNA was synthesized using a PrimeScript RT Reagent Kit (TaKaRa) according to the manufacturer's instructions. Quantitative real-time PCR was carried out using SYBR Premix Ex Taq (TaKaRa) to determine the expression level of gene transcripts for miR-214-5p, U6, E-cadherin, N-cadherin, Vimentin, and $\beta$-actin. The sequences of real-time PCR primers were as follows:

WASL forward 5' - GAACGAGTCCCTCTTCACTTTC - $3^{\prime}$; reverse 5'- GTTCCGATCTGCTGCATATAACT -3; E-cadherinforward $5^{\prime}$-GGACAGCCTATTTTTCCCTCGAC- 3 '; reverse $5^{\prime}$-ACCCAGTCTCTCTTCTGTCTTCT-3; N-cadherinforward $5^{\prime}$-ATCCCTCCAATCAACTTGCCAGA-3'; ${ }^{\text {; }}$ - 2 verse $5^{\prime}$-CCCATTCCAAACCTGGTGTAAGA-3; Vimentin forward 5' - CAGGACTCGGTGGACTTCTCGCT-3'; reverse 5'- TTCTCCCGGAGGCGCATGATGTC-3; $\beta$-actin forward 5' - ATCCAGGCTGTGCTGTCCCCGTA-3'; reverse 5'- GAAGTCCAGGGCAACATAGCACA-3;

Quantitative PCR was conducted in triplicate at $95^{\circ} \mathrm{C}$ for $10 \mathrm{~min}$, followed by 40 cycles of $95^{\circ} \mathrm{C}$ for $15 \mathrm{~s}$ and $60^{\circ} \mathrm{C}$ for $60 \mathrm{~s}$ (7300 Fast Real-Time PCR System;Stratagen). Cycle thresholds were normalized to an internal control: U6 for precursor of miRNA and $\beta$-actin for mRNA assays. The amount of RNA was calculated using the $2^{-\Delta \Delta C T}$ method; the level of expression of an RNA was normalized to the adapted internal control (denoted "relative expression").

\section{Western blot}

The total protein of cells and tissues was extracted with RIPA lysis solution (Beyotime biotechnology, USA) containing PMSF 1\%; the protein concentration was detected by BCA protein analysis kit. After $10 \%$ sodium dodecyl sulfate-polyacrylamide gel electrophoresis (SDS-PAGE), the protein was transferred onto PVDF membrane and then incubated for $1 \mathrm{~h}, 300 \mathrm{~mA}$. Blocked with Tris-buffered saline and Tween (TBST) containing $5 \%$ skim milk. Using $\beta$-actin as an internal reference, the membrane was subsequently incubated at $4^{\circ} \mathrm{C}$ overnight with primary antibodies against WASL, E-cadherin, N-cadherin, Vimentin diluted 1:1000 (Santa Cruz Biotechnology, Inc., Santa Cruz, CA, USA) and $\beta$-actin diluted 1:5000 (Santa 
Cruz Biotechnology, Inc., Santa Cruz, CA, USA). Next, the membranes were incubated with horseradish peroxidase (HRP)-labeled secondary antibodies at room temperature for $2 \mathrm{~h}$, followed ECL. The image analysis software Gel-Pro Analyzer 4.0 was used to analyze the gray scale images. The relative ratio was calculated by a comparison with the expression of the internal reference gene. The experiment was conducted 3 times for each group.

\section{Results}

miR-214-5p is significantly decreased in HCC

To explore whether the expression of miR-214-5p has difference between hepatocellular carcinoma tissue and para-carcinoma tissue. We collected 44 pairs of hepatocellular carcinoma and para-carcinoma tissues from the Fist affiliated hospital of Jinzhou medical university. By qRT-PCR, we found the expression of miR-214-5p was significantly lower in HCC tissues than in adjacent normal tissues $(P<0.05)$. Moreover, to further explore whether miR-214-5p was lower expressed in cancer cell lines compared to a normal hepatic cell line $[23,24]$, we detected the expression of miR-214-5p in cell lines. The results showed that miR214-5p was significantly lower in Hepatic cell lines (HepG2, BEL-7402) than in normal hepatic cell line ( Chang liver cells) (Fig. 1).

Overexpression of miR-214-5p suppressed invasion and migration of HCC

To detect the role of miR-214-5p in HCC cell invasion and migration. HepG2 and BEL-7402 cells were transfected with miR-214-5p mimic, miR-214-5p inhibitor and corresponding controls. qRT-PCR showed that the expression level of miR-214-5p was significantly

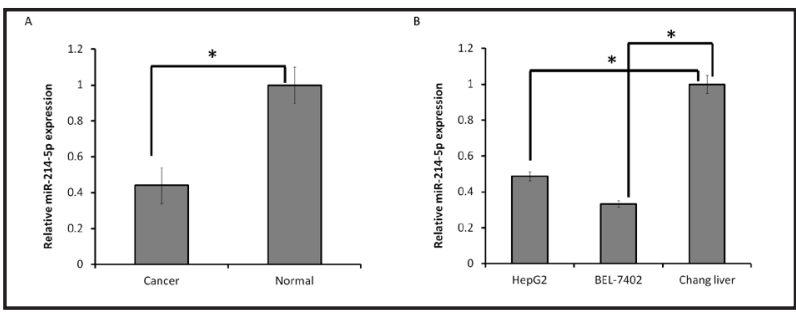

Fig. 1. A: Expression of miR-214-5p in HCC tissues and normal hepatic tissues. qRT-PCR analysis of miR-214-5p levels in 44 pairs of hepatocellular carcinoma and paracarcinoma tissues. Normal, para-carcinoma tissue. Cancer, hepatocellular carcinoma. *: P<0.05; B: miR-214-5p was significantly lower in HepG2 and BEL-7402 than in Chang liver cells. *: $\mathrm{P}<0.05$.

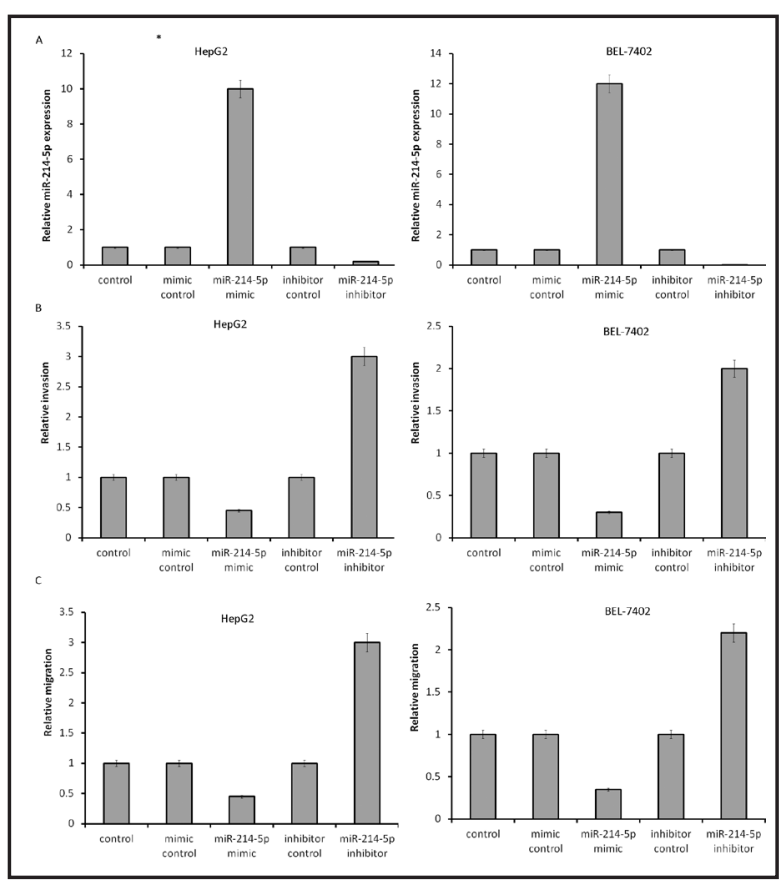

Fig. 2. miR-214-5p inhibited cell invasion and migration. HepG2 and BEL-7402 were transfected with miR-214-5p mimic, mimic control, miR-214-5p inhibitor and inhibitor control. A: miR-214-5p expression was up-regulated in miR-214-5p mimic transfected cells but down-regulated in miR-214-5p inhibitor transfected cells; B: Overexpression of miR-214-5p inhibited cell invasion, but supression of miR-214-5p promoted invasion in HepG2 and BEL7402 cells; C: Overexpression of miR-214-5p inhibited cell migration, but supression of miR-214-5p promoted migration in HepG2 and BEL-7402 cells. up-regulated by miR-214-5p mimic and down-regulated by miR-214-5p inhibitor (Fig. 2A). In terms of cell migration and invasion, miR-214-5p overexpression remarkably decreased cells migration and invasion in HepG2 and BEL-7402 cells compared to its control group. Meanwhile, these effects were reversed by miR-214-5p suppression (Fig. 2B-2C). 


\begin{abstract}
miR-214-5p suppressed EMT in HCC cells

To explore the effect of miR-214-5p on EMT process, HepG2 and BEL7402 were transfected with miR-214-5p mimic, miR-214-5p inhibitor and corresponding controls. The protein levels of EMT-related factors were detected by western blot. The results showed that miR-214-5p promoted E-cadherin expression but inhibited $\mathrm{N}$-cadherin, Vimentin expression (Fig. $3)$.
\end{abstract}

WASL was a putative target of $m i R-214-5 p$

To explore the target gene of miR-214-5p, the biological information online analysis softwares (TargetScan, miRDB, and microrna) were used. WASL was confirmed to be a target gene of miR-214-5p. The luciferase reporter vector, which contained WASL 3'UTR fragments with wildtype or mutant, miR-214$5 p$ complementary sites, is shown in Fig. 4A. The dualluciferase reporter assay (Fig. 4B) demonstrated a significant difference in the luciferase activity among the miR-214-5p-NC + WASL-3'UTR, miR-214-5p-NC + WASL-3' UTR mut (the WASL3'UTR was deleted), miR-214-5p mimics + WASL-3' UTR and miR214-5p mimics + WASL-3' UTR mut groups $(P<0.001)$. Compared with the miR-214-5p-NC + WASL-

3'UTR, miR-214-5p-NC + WASL-3' UTR mut and miR-214-5p mimics + WASL-3'UTR mut groups, the luciferase activity was significantly decreased in the miR-214-5p mimics + WASL3'UTR group $(P<0.05)$. qRT-PCR revealed a significant difference in miR-214-5p expression among the four groups $(P<0.001)$. The expression of miR-214-5p mimics + WASL-3' UTR mut groups compared with the miR-214-5p-NC + WASL-3'UTR and miR-214-5p-NC + WASL3 ' UTR mut groups (all $P<0.05$ ). To explore the relationship between miR-214-5p and WASL, HepG2 was transfected with miR-214-5p mimic, miR-214-5p inhibitor and corresponding controls. qRT-PCR and western blot assays were used to examine the mRNA and protein levels of WASL in different groups. The results displayed that mRNA expression of WASL were

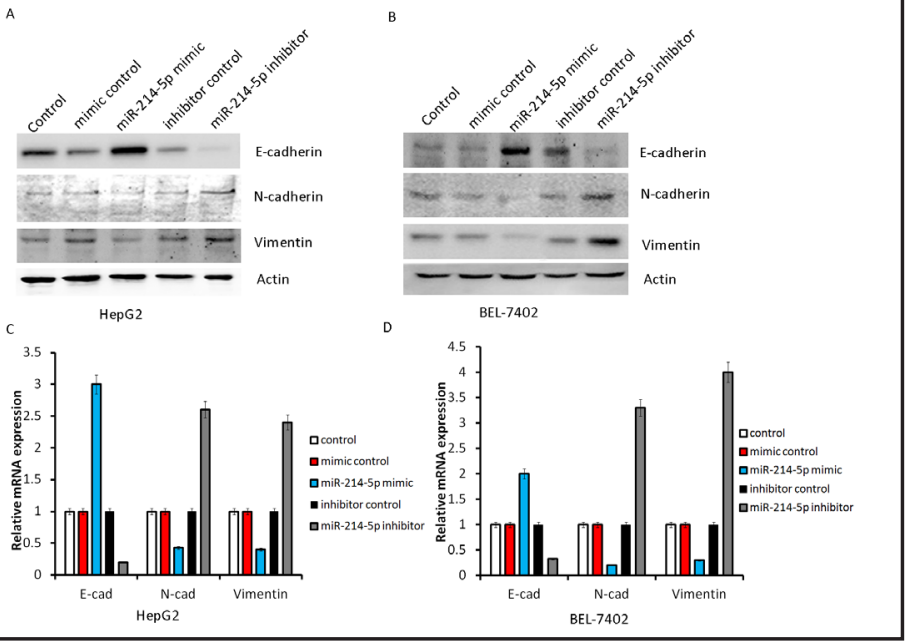

Fig. 3. miR-214-5p inhibited EMT process. HepG2 and BEL-7402 cells were transfected with miR-214-5p mimic, miR-214-5p inhibitor and corresponding controls. A and B: Western blot showed overexpression of miR-214-5p promoted E-cadherin pression but inhibited N-cadherin, Vimentin expressions in HepG2 and BEL-7402 cells. C and D: qRT-PCR showed overexpression of miR-214-5p promoted E-cadherin pression but inhibited $\mathrm{N}$-cadherin, Vimentin expressions in HepG2 and BEL7402 cells.

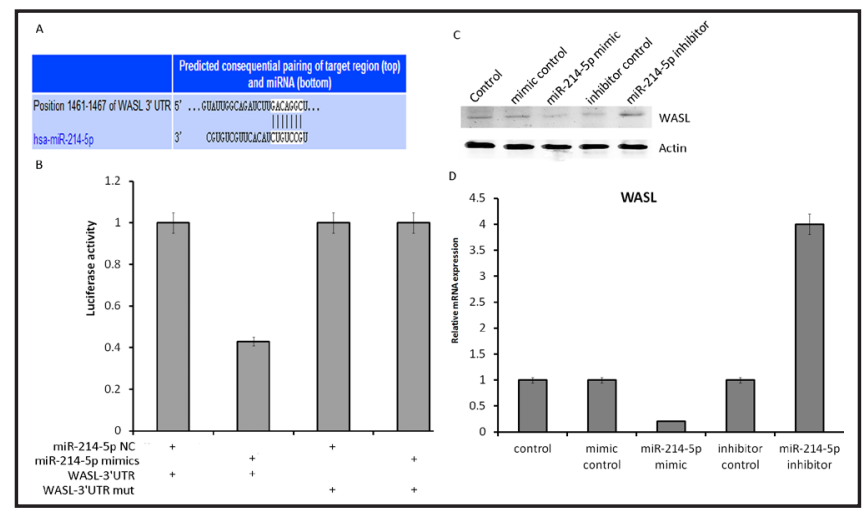

Fig. 4. WASL was a target of miR-214-5p. A: Schematic diagram of putative binding sites of miR-214-5p in the 3'-UTR of WASL; B: Dual-luciferase reporter assay showed WASL is the target genes of miR-214-5p in human HepG2 cells; C and D: miR-214$5 p$ negatively regulated the mRNA and protein expression of WASL in HepG2 cells. 
Fig. 5. miR-214-5p inhibit cell invasion and migration by regulating WASL. A: Invasion ability of HepG2 and BEL-7402 cells $48 \mathrm{~h}$ after transfection was detected by Transwell chamber assay in each group; B: migration ability of HepG2 and BEL-7402 cells $48 \mathrm{~h}$ after transfection was detected by Transwell chamber assay in each group.

signally down-regulated by miR-214-5p overexpression but up-regulated by miR214-5p inhibitor compared to corresponding controls (Fig. 4D). Western blot analysis with a similar trend to mRNA level of WASL (Fig. 4C).

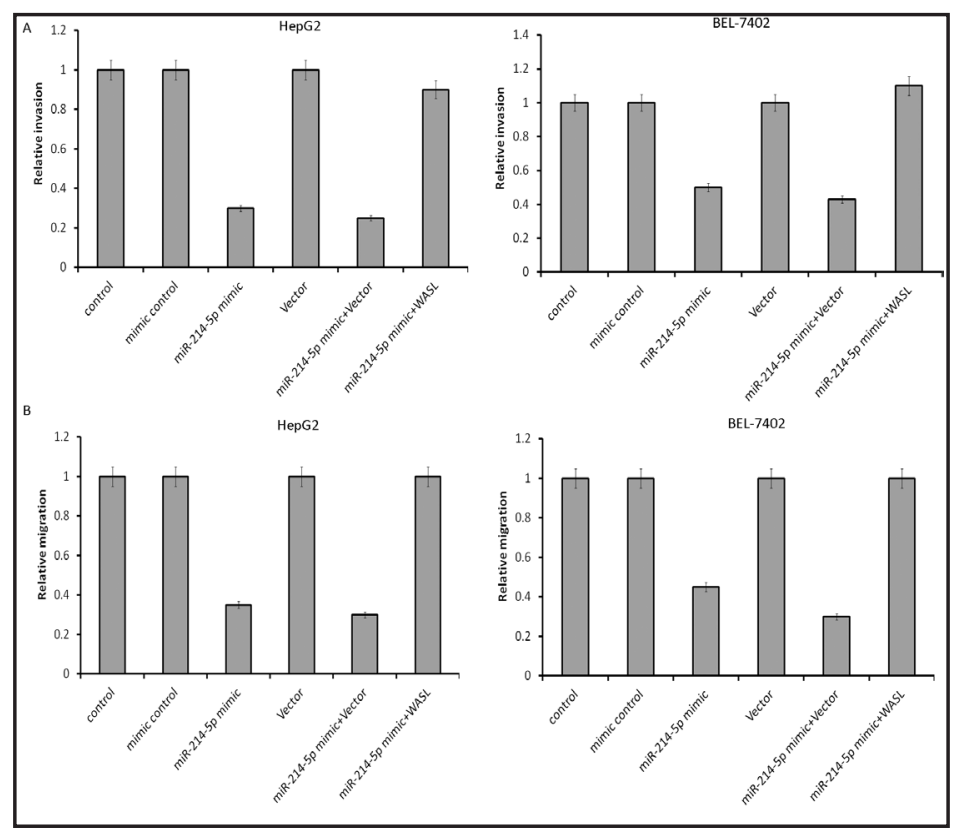

miR-214-5p inhibit cell invasion and migration by regulating WASL

As shown by the invasion and migration assay (Fig. 5), remarkable significant differences were found in the invasion ability of HCC cells among the different groups $48 \mathrm{~h}$ after transfection $(P<0.001)$. Compared with the control, mimic control, Vector and miR214-5p mimics + WASL groups, the invasion ability of cells in the miR-214-5p mimics and miR-214-5p mimics + Vector group were significantly decreased $(P<0.05)$. No significant difference was seen in the invasion ability of cells among the control, mimic control, Vector and miR-214-5p mimics + WASL groups (all $P>0.05$ ).

\section{Discussion}

Invasion and metastasis has been well acknowledged as one of acquired capacities of cancer cells [25]. The exceedingly complex process involved many molecular mechanisms [26-28]. Among them, the proteins of the WASP family regulate Arp2/3-related pathway [2931]. WASL, which belongs to WASP family, was found to be expressed in colorectal cancer liver metastases and esophageal squamous cell carcinoma [32-34]. WASL and WAVE-2 expression were shown to be extensive in HCC [35]. Researchers try to regulate the expression of WASP family proteins to inhibit the invasion of cancer cells [36-38]. Among them, microRNAs gain many attention for their function as gene expression regulators. Just recently, miR-214-5p has been found to influence tumor process [15]. However, the effect of miR-214-5p on HCC remains unknown.

In the present study, we found, by qRT-PCR that miR-214-5p was low expressed in HCC tissues compared with parental tissues, indicating that miR-214-5p might be involved in carcinogenesis of HCC. MiR-214-5p is lower expressed in cancer tissues than parental tissues in 44 pairs of HCC. Additionally, we overexpressed miR-214-5p in HepG2 and BEL-7402 cells and found miR-214-5p suppressed invasion and migration of HCC. Not only that, miR-214$5 p$ also suppressed EMT, promoted expression of E-cadherin, and inhibited the expression of N-cadherin and Vimentin. By bioinformation software, we found WASL was a putative target of miR-214-5p. Then, we examed the correlation between miR-214-5p and WASL, and the results showed miR-214-5p could down-regulate the expression of WASL by qRT-PCR and Western blot. Dual-luciferase reporter gene analysis showed WASL is the target gene of miR-214-5p. The over-expression of miR-214-5p can inhibit invasion and migration of HCC, and targeting WASL activates this process. So, up-regulating the expression of WASL could 


\section{Cellular Physiology Cell Physiol Biochem 2018:46:757-764 \begin{tabular}{l|l} 
DOI: 10.1159/000488734 & $\begin{array}{l}\text { O 2018 The Author(s). Published by S. Karger AG, Basel } \\
\text { www.karger.com/cpb }\end{array}$
\end{tabular}}

Li et al.: miR-214-5p Inhibits Hepatocellular Carcinoma

reverse the inhibition effect of miR-214-5p on HCC. These data indicated that miR-214-5p could inhibit invasion and migration through targeting WASL in HCC.

Taken together, our study demonstrated that miR-214-5p as a tumor suppressor that inhibited HCC cells invasion and migration and blocked the process by targeting WASL. These findings suggested that miR-214-5p might be considered as a putative target in the treatment of Hepatocellular carcinoma.

\section{Acknowledgments}

This work was supported by the grants from National Natural Science Foundation of China (81502484) and the project of innovation and entrepreneurship training program for undergraduate of Liaoning province (201610160000053).

\section{Disclosure Statement}

No conflict of interests exists.

\section{References}

1 Parkin DM, Bray F, Ferlay J, Pisani P: Global cancer statistics, 2002. CA Cancer J Clin 2005;55:74-108.

$>2$ Jemal A, Siegel R, Ward E, Murray T, Xu J, Thun MJ: Cancer statistics, 2007. CA Cancer J Clin 2007;57:43-66.

-3 Poon RT, Fan ST, Lo CM, Liu CL, Wong J: Intrahepatic recurrence after curative resection of hepatocellular carcinoma: long-term results of treatment and prognostic factors. Ann Surg 1999;229:216-222.

4 Minagawa M, Makuuchi M, Takayama T, Kokudo N: Selection criteria for repeat hepatectomy in patients with recurrent hepatocellular carcinoma. Ann Surg 2003;238:703-710.

5 Llovet JM, Burroughs A, Bruix J: Hepatocellular carcinoma. Lancet 2003;362:1907-1917.

6 Zhu YB, Xu X, Zheng SS: [Association of microvascular invasion with recurrence and prognosis of patients with small hepatocellular carcinoma undergoing liver transplantation]. Zhejiang Da Xue Xue Bao Yi Xue Ban 2014;43:658-663.

7 Sui J, Yang X, Qi W, Guo K, Gao Z, Wang L, Sun D: Long Non-Coding RNA Linc-USP16 Functions As a Tumour Suppressor in Hepatocellular Carcinoma by Regulating PTEN Expression. Cell Physiol Biochem 2017;44:1188-1198.

-8 Bie B, Sun J, Li J, Guo Y, Jiang W, Huang C, Yang J, Li Z: Baicalein, a Natural Anti-Cancer Compound, Alters MicroRNA Expression Profiles in Bel-7402 Human Hepatocellular Carcinoma Cells. Cell Physiol Biochem 2017;41:1519-1531.

-9 Sanchez AM, Flamini MI, Baldacci C, Goglia L, Genazzani AR, Simoncini T: Estrogen receptor-alpha promotes breast cancer cell motility and invasion via focal adhesion kinase and N-WASP. Mol Endocrinol 2010;24:2114-2125.

10 Liu Y, Chai Y, Zhang J, Tang J: A Function Variant at miR-501 Alters Susceptibility to Hepatocellular Carcinoma in a Chinese Han Population. Cell Physiol Biochem 2016;38:2500-2508.

11 Sun JJ, Chen GY, Xie ZT: MicroRNA-361-5p Inhibits Cancer Cell Growth by Targeting CXCR6 in Hepatocellular Carcinoma. Cell Physiol Biochem 2016;38:777-785.

12 Lee YB, Bantounas I, Lee DY, Phylactou L, Caldwell MA, Uney JB: Twist-1 regulates the miR-199a/214 cluster during development. Nucleic Acids Res 2009;37:123-128.

13 Iizuka M, Ogawa T, Enomoto M, Motoyama H, Yoshizato K, Ikeda K, Kawada N: Induction of microRNA-2145p in human and rodent liver fibrosis. Fibrogenesis Tissue Repair 2012;5:12.

-14 Elemeery MN, Badr AN, Mohamed MA, Ghareeb DA: Validation of a serum microRNA panel as biomarkers for early diagnosis of hepatocellular carcinoma post-hepatitis C infection in Egyptian patients. World J Gastroenterol 2017;23:3864-3875.

15 Zhang M, Wang D, Zhu T, Yin R: miR-214-5p Targets ROCK1 and Suppresses Proliferation and Invasion of Human Osteosarcoma Cells. Oncol Res 2017;25:75-81.

$>16$ Li QS, Meng FY, Zhao YH, Jin CL, Tian J, Yi XJ: Inhibition of microRNA-214-5p promotes cell survival and extracellular matrix formation by targeting collagen type IV alpha 1 in osteoblastic MC3T3-E1 cells. Bone Joint Res 2017;6:464-471. 


\section{Cellular Physiology Cell Physiol Biochem 2018;46:757-764 \begin{tabular}{l|l} 
DOI: 10.1159/000488734 & $\begin{array}{l}\text { O } 2018 \text { The Author(s). Published by S. Karger AG, Basel } \\
\text { www.karger.com/cpb }\end{array}$ \\
\hline
\end{tabular}}

Li et al.: miR-214-5p Inhibits Hepatocellular Carcinoma

17 Chandrasekaran KS, Sathyanarayanan A, Karunagaran D: MicroRNA-214 suppresses growth, migration and invasion through a novel target, high mobility group AT-hook 1, in human cervical and colorectal cancer cells. Br J Cancer 2016;115:741-751.

18 Yang Y, Zhao Z, Hou N, Li Y, Wang X, Wu F, Sun R, Han J, Sun H, Song T, Huang C, Shao Y: MicroRNA214 targets Wnt3a to suppress liver cancer cell proliferation. Mol Med Rep 2017;16:6920-6927.

19 Li Y, Li Y, Chen Y, Xie Q Dong N, Gao Y, Deng H, Lu C, Wang S: MicroRNA-214-3p inhibits proliferation and cell cycle progression by targeting MELK in hepatocellular carcinoma and correlates cancer prognosis. Cancer Cell Int 2017;17:102.

-20 Li HL, Liang S, Cui JH, Han GY: Targeting of GSK-3beta by miR-214 to facilitate gastric cancer cell proliferation and decrease of cell apoptosis. Eur Rev Med Pharmacol Sci 2018;22:127-134.

-21 Yang L, Zhang W, Wang Y, Zou T, Zhang B, Xu Y, Pang T, Hu Q Chen M, Wang L, Lv Y, Yin K, Liang H, Chen X, Xu G, Zou X: Hypoxia-induced miR-214 expression promotes tumour cell proliferation and migration by enhancing the Warburg effect in gastric carcinoma cells. Cancer Lett 2018;414:44-56.

-22 Zhang Q, Zhang S: miR-214 promotes radioresistance in human ovarian cancer cells by targeting PETN. Biosci Rep 2017;37:

-23 Xu J, Zhu X, Wu L, Yang R, Yang Z, Wang Q, Wu F: MicroRNA-122 suppresses cell proliferation and induces cell apoptosis in hepatocellular carcinoma by directly targeting Wnt/beta-catenin pathway. Liver Int 2012;32:752-760.

-24 Yoon SY, Jeong MJ, Yoo J, Lee KI, Kwon BM, Lim DS, Lee CE, Park YM, Han MY: Grb2 dominantly associates with dynamin II in human hepatocellular carcinoma HepG2 cells. J Cell Biochem 2001;84:150-155.

25 Hanahan D, Weinberg RA: The hallmarks of cancer. Cell 2000;100:57-70.

26 Yamaguchi H, Wyckoff J, Condeelis J: Cell migration in tumors. Curr Opin Cell Biol 2005;17:559-564.

27 Yamazaki D, Kurisu S, Takenawa T: Regulation of cancer cell motility through actin reorganization. Cancer Sci 2005;96:379-386.

-28 Gassmann P, Enns A, Haier J: Role of tumor cell adhesion and migration in organ-specific metastasis formation. Onkologie 2004;27:577-582.

29 Rohatgi R, Ma L, Miki H, Lopez M, Kirchhausen T, Takenawa T, Kirschner MW: The interaction between N-WASP and the Arp2/3 complex links Cdc42-dependent signals to actin assembly. Cell 1999;97:221-231.

30 Yamaguchi H, Miki H, Takenawa T: Neural Wiskott-Aldrich syndrome protein is involved in hepatocyte growth factor-induced migration, invasion, and tubulogenesis of epithelial cells. Cancer Res 2002;62:25032509.

-31 Kawamura K, Takano K, Suetsugu S, Kurisu S, Yamazaki D, Miki H, Takenawa T, Endo T: N-WASP and WAVE2 acting downstream of phosphatidylinositol 3-kinase are required for myogenic cell migration induced by hepatocyte growth factor. J Biol Chem 2004;279:54862-54871.

-32 Yanagawa R, Furukawa Y, Tsunoda T, Kitahara O, Kameyama M, Murata K, Ishikawa O, Nakamura Y: Genome-wide screening of genes showing altered expression in liver metastases of human colorectal cancers by cDNA microarray. Neoplasia 2001;3:395-401.

-33 Wang WS, Zhong HJ, Xiao DW, Huang X, Liao LD, Xie ZF, Xu XE, Shen ZY, Xu LY, Li EM: The expression of CFL1 and N-WASP in esophageal squamous cell carcinoma and its correlation with clinicopathological features. Dis Esophagus 2010;23:512-521.

-34 Yang LY, Tao YM, Ou DP, Wang W, Chang ZG, Wu F: Increased expression of Wiskott-Aldrich syndrome protein family verprolin-homologous protein 2 correlated with poor prognosis of hepatocellular carcinoma. Clin Cancer Res 2006;12:5673-5679.

-35 Shim JH, Yoon DL, Han S, Lee YJ, Lee SG, Kim KM, Lim YS, Lee HC, Chung YH, Lee YS: Is serum alphafetoprotein useful for predicting recurrence and mortality specific to hepatocellular carcinoma after hepatectomy? A test based on propensity scores and competing risks analysis. Ann Surg Oncol 2012;19:3687-3696.

-36 Frugtniet B, Jiang WG, Martin TA: Role of the WASP and WAVE family proteins in breast cancer invasion and metastasis. Breast Cancer (Dove Med Press) 2015;7:99-109.

-37 Kurisu S, Takenawa T: WASP and WAVE family proteins: friends or foes in cancer invasion? Cancer Sci 2010;101:2093-2104.

-38 Hou J, Yang H, Huang X, Leng X, Zhou F, Xie C, Zhou Y, Xu Y: N-WASP promotes invasion and migration of cervical cancer cells through regulating p38 MAPKs signaling pathway. Am J Transl Res 2017;9:403-415. 